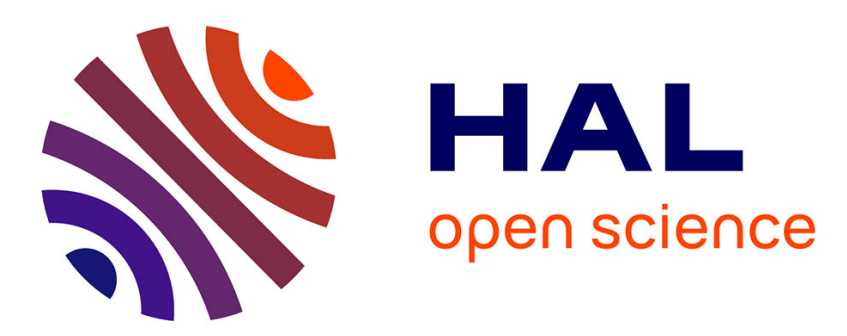

\title{
Le sperme des salmonidés: le point sur les connaissances. Applications à la salmoniculture
}

Gérard Maisse

\section{To cite this version:}

Gérard Maisse. Le sperme des salmonidés : le point sur les connaissances. Applications à la salmoniculture. Productions Animales, 1990, 3 (3), pp.223-228. 10.20870/productions-animales.1990.3.3.4379 . hal-00895906

\section{HAL Id: hal-00895906 https://hal.science/hal-00895906}

Submitted on 1 Jan 1990

HAL is a multi-disciplinary open access archive for the deposit and dissemination of scientific research documents, whether they are published or not. The documents may come from teaching and research institutions in France or abroad, or from public or private research centers.
L'archive ouverte pluridisciplinaire HAL, est destinée au dépôt et à la diffusion de documents scientifiques de niveau recherche, publiés ou non, émanant des établissements d'enseignement et de recherche français ou étrangers, des laboratoires publics ou privés. 
INRA Prod. Anim., 1990, $3(3), 223-228$

\section{G. MAISSE}

INRA Laboratoire de Physiologie des Poissons

35042 Rennes cedex
Le sperme des salmonidés : le point sur les connaissances. Applications à la salmoniculture.

S'appuyant depuis l'origine exclusivement sur la fécondation artificielle in vitro, la salmoniculture est tributaire d'une bonne gestion des gamètes, et en particulier du sperme. Une bonne connaissance des caractéristiques ultrastructurales et physiologiques des spermatozoïdes de salmonidés permet de proposer des méthodes pour les manipuler, les conserver et les congeler.

La salmoniculture est l'élevage des poissons de la famille des salmonidés qui comprend trois genres: Salmo (truite fario et saumon atlantique), Salvelinus (ombles) et Oncorhynchus (truite arc-en-ciel, saumons du pacifique). En France l'essentiel de la production concerne la truite arc-en-ciel (environ 30000 tonnes) introduite d'Amérique du Nord au début du siècle.

La salmoniculture est née avec la redécouverte de la fécondation artificielle, au milieu du 19 ème siècle. Les premiers traités de pisciculture (Coste 1856) montrent déjà l'importance de la gestion et de la manipulation des gamètes, dans un élevage où l'on pratique, dès l'origine, la fécondation in vitro. Aujourd'hui, cette importance s'est accrue avec l'apparition de nouveaux modes de gestion des géniteurs et des gamètes qui demandent de la part du pisciculteur plus de technicité et de rigueur: le contrôle photopériodique de la date de ponte, l'utilisation de «néomâles» (génétiquement

\section{Résumé}

Une revue d'un certain nombre de travaux portant sur le sperme de salmonidé permet d'en dégager les particularités : l'absence d'acrosome, l'immobilité dans le fluide séminal liée à la présence de l'ion potassium dont la dilution est la première condition de l'activation, la faible durée de la motilité qui est due à l'incapacité de la mitochondrie à compenser rapidement les pertes en ATP, la dépendance vis à vis des conditions de milieu (température, $\mathrm{pH}$, oxygène).

Ces caractéristiques entraînent un certain nombre de recommandations au niveau de la pratique piscicole, et la mise au point de dilueurs de fécondation, de conservation à court terme et de congélation. femelles) pour la production de cohortes «monosexe-femelle " et la triploïdisation pour la production de grandes truites stériles sont devenus des outils indispensables à l'élevage industriel, mais, relevant du domaine biologique, ils peuvent être source d'échec en cas de non respect de règles élémentaires.

Nous présentons ici un certain nombre de données qui nous paraissent essentielles à la bonne compréhension des techniques mises en oeuvre pour la manipulation et la conservation du sperme de salmonidés.

\section{1 / Caractéristiques générales du sperme}

Les salmonidés sont des poissons à reproduction saisonnière: en France, la truite fario et le saumon atlantique, les seuls représentants indigènes de la famille dans les rivières, se reproduisent de novembre à février; en pisciculture, les souches de truite arc-en-ciel les plus couramment élevées donnent des pontes depuis début octobre jusqu'à fin mars. Les oeufs de chaque femelle sont prélevés dans leur totalité en une seule fois ; les mâles sont spermiants pendant deux à trois mois et peuvent fournir du sperme plusieurs fois.

Le sperme des salmonidés est un liquide blanc laiteux, couleur qui lui a valu la dénomination de laitance employée par les auteurs anciens (Coste 1856). Le volume d'un éjaculat, obtenu par pression des flancs d'un mâle mature, varie avec la taille de l'individu et avec l'avancement de la période de spermiation ; il peut dépasser $50 \mathrm{ml}$. Le nombre de spermato- 
zoïdes par millilitre d'éjaculat varie de $10^{9}$ à 30 x $10^{\mathrm{s}}$, la moyenne se situant aux environs de 10 $x 10^{\circ}$. Sur l'ensemble d'une saison de reproduction un mâle de $1 \mathrm{~kg}$, prélevé hebdomadairement, peut donner jusqu'à $10^{12}$ spermatozoïdes (Maisse et al 1988).

Le fluide séminal, dans lequel baignent les spermatozoïdes, représente plus de $80 \%$ du volume du sperme (Munkittrick et Moccia 1987). Son $\mathrm{pH}$ varie suivant les auteurs de 7,3 à 8,3 (Baynes et al 1981). Son osmolarité est variable d'un éjaculat à l'autre pour le même animal (de moins de 100 à plus de $300 \mathrm{mOs}$ moles, Maisse et al 1988). Les principaux ions sont $\mathrm{Na}^{+}, \mathrm{K}^{+}, \mathrm{Ca}^{+}, \mathrm{Mg}^{+}$et $\mathrm{Cl}^{-}$; les compositions ioniques rapportées dans la littérature varient selon les auteurs, les espèces et, au cours de la saison de reproduction, en rapport avec la densité en spermatozoïdes (Piironen 1985). Les valeurs rapportées par Morisawa (1985) (tableau 1), montrent, que chez les salmonidés, le fluide séminal se distingue du plasma sanguin par une teneur en potassium très nettement supérieure, l'osmolarité étant équivalente; d'après les travaux in vitro de Marshall et al (1989) c'est l'hormone gonadotrope qui stimulerait l'absorption de $\mathrm{Na}$ et la secrétion de $\mathrm{K}^{*}$ par l'épithélium du canal déférent.

Tableau 1. Composition ionique (en $m M$ ) et osmolarité (en MOsmoles) du fluide séminal et du plasma sanguin de la truite arc-en-ciel (d'après Morisawa 1985).

\begin{tabular}{|ccc|}
\hline & $\begin{array}{c}\text { fluide } \\
\text { séminal }\end{array}$ & $\begin{array}{c}\text { plasma } \\
\text { sanguin }\end{array}$ \\
\hline $\mathrm{Na}^{+}$ & 127 & 146 \\
$\mathrm{~K}^{+}$ & 37 & 3,1 \\
$\mathrm{Ca}^{++}$ & 2,6 & 3,3 \\
$\mathrm{Mg}^{++}$ & 1,5 & 0,9 \\
$\mathrm{Cl}^{-}$ & 122 & $\mathbf{1 1 5}$ \\
\hline osmolarité & 297 & 292 \\
\hline
\end{tabular}

De nombreux acides aminés sont présents dans le fluide séminal, mais leur niveau est 10 fois moindre que dans le sang (Billard et Menezo 1984) ; c'est la taurine qui est l'acide aminé le mieux représenté. La présence de ces acides aminés pourrait être une composante non négligeable de la pression osmotique, comme cela est le cas chez la carpe où la teneur séminale est 4 fois plus importante que celle du sang (Billard et Menezo 1984).

La concentration en protéines est 20 fois moindre dans le fluide séminal que dans le sang (Loir et al 1990). De nombreuses protéines ont une parenté immunologique avec des protéines sanguines; elles peuvent provenir du sang, mais il n'est pas exclu que certaines soient néo-synthétisées par les cellules de Sertoli et/ou les.cellules du canal déférent; leurs poids moléculaires varient de $11 \mathrm{KDa}$ à $80 \mathrm{KDa}$. D'autres protéines proviennent des spermatozoïdes eux-mêmes avec en particulier la $42 \mathrm{KDa}$ qui est un des constituants majeurs de la membrane et la $49 \mathrm{KDa}$ qui domine dans les pro- téines flagellaires. Une quantité plus ou moins importante de $42 \mathrm{KDa}$ dans le liquide séminal est interprétée comme le témoignage d'une plus ou moins grande dégradation de la membrane des spermatozoïdes (Maisse et al 1988). Des lipoprotéines (HDL) en faible quantité ont été décrites et pourraient jouer un rôle dans la conservation de la membrane dans le canal déférent (Loir et al 1990).

\section{2 / Ultrastructure du spermatozoïde}

Le spermatozoïde de truite (Billard 1983, figure 1) est constitué d'une tête de $2,5 \mu \mathrm{m}$ de long pour 1,5 à $2 \mu \mathrm{m}$ de diamètre (ce qui correspond au diamètre du micropyle de l'œuf par où il pénètre au moment de la fécondation et, de ce fait, limite le phénomène de polyandrie) et d'un flagelle de 25 à $35 \mu \mathrm{m}$ de long. La tête, occupée essentiellement par le noyau, est dépourvue d'acrosome. La pièce intermédiaire renferme une mitochondrie annulaire non fermée, dans un cytoplasme très réduit. L'axonème, inséré sur le centriole distal dans une dépression de la base du noyau, comprend 9 doublets de microtubules périphériques et 2 microtubules centraux. La membrane flagellaire présente 2 extensions cytoplasmiques latérales dans lesquelles des vésicules sont visibles.

Figure 1. Ultra structure du spermatozoïde de truite arc-en-ciel (d'après Billard 1983).

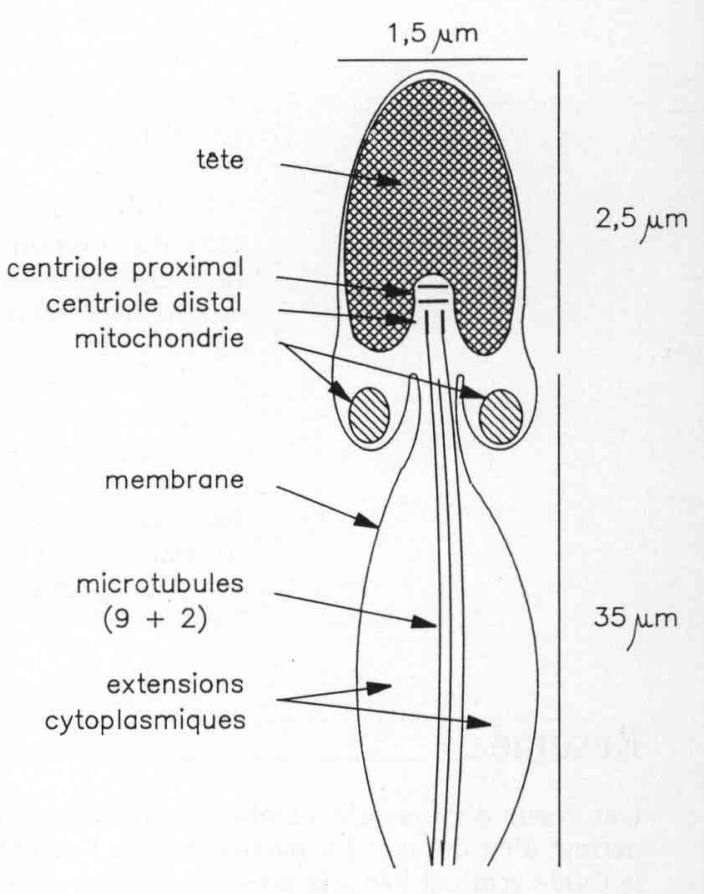

\section{3 / Physiologie du spermatozoïde}

\section{1 / La respiration}

La consommation d'oxygène des spermatozoïdes immobiles augmente avec la tempéra- 
Figure 2. Consommation en oxygène des spermatozoïdes (valeurs rapportées pour $15.109 \mathrm{cel}-$ lules) de truite immobiles, en fonction de la température (d'après Robitaille et al 1987).

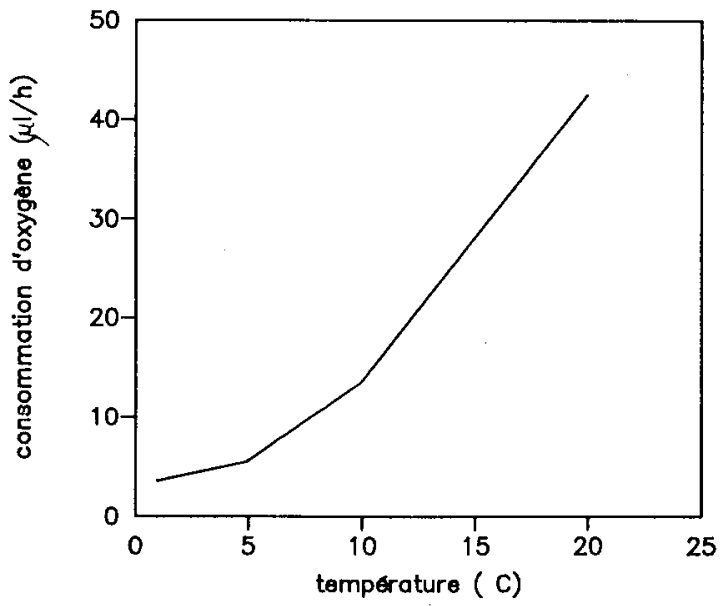

ture (Robitaille et al 1986, figure 2 ; à $10^{\circ} \mathrm{C}$ la demande en $\mathrm{O} 2$ est d'environ $10 \mu \mathrm{l} /$ heure pour $10^{10}$ spermatozoïdes.

\section{2 / L'osmorégulation}

La concentration des cations, $\mathrm{Na}^{+}, \mathrm{K}^{+}, \mathrm{Mg}^{++}$ et $\mathrm{Ca}^{++}$a été étudiée pour le spermatozoïde de Saumon atlantique par Hwang et Idler (1969) (tableau 2) ; l'observation de gradients inverses en sodium et en potassium de part et d'autre de la membrane suggèrent l'existence d'un transport actif mettant en jeu la $\mathrm{Na}^{+}-\mathrm{K}^{+}$, ATPase. Par ailleurs, dans une solution hypotonique le spermatozoïde se comporte comme un osmomètre, l'entrée d'eau provoque le gonflement, voire l'éclatement, de la membrane (Maléjac et al 1990), ce phénomène existe lorsque la fécondation a lieu dans de l'eau douce, c'est un des facteurs limitants pour l'obtention de résultats constants avec du sperme fortement dilué.

Tableau 2. Composition ionique (en $m g / 100 \mathrm{~g}$ ) du fluide séminal et du spermatozoïde de saumon atlantique (d'après Hwang et Idler 1969).

\begin{tabular}{|ccc|}
\hline & fluide séminal & spermatozoïde \\
\hline $\mathrm{Na}^{+}$ & 237 & 84 \\
$\mathrm{~K}^{+}$ & 86 & 298 \\
$\mathrm{Ca}^{++}$ & 5,2 & 1,5 \\
$\mathrm{Mg}^{++}$ & 2,2 & 18,7 \\
\hline
\end{tabular}

\section{3 / La motilité}

\section{a / Description}

La motilité des spermatozoïdes peut être étudiée d'une manière globale, à partir d'une table de notation permettant l'interprétation des observations réalisées au microscope (SanchezRodriguez et Billard 1977, tableau 3) ou de manière plus précise à l'aide de la stroboscopie (Cosson et al 1985) qui permet de détermị̣er la fréquence de battement du flagelle.
Dans une solution saline, le mouvement d'un spermatozoïde isolé suit une trajectoire en spirale avec une vitesse de $250 \mu \mathrm{m} / \mathrm{s}$ après $5 \mathrm{~s}$ et de $50 \mu \mathrm{m} / \mathrm{s}$ au bout de $20 \mathrm{~s}$ (Gatti et al 1985). Dans les mêmes conditions, la fréquence initiale de battement du flagelle dépend de la température avec un maximum voisin de $60 \mathrm{~Hz}$ pour $20^{\circ} \mathrm{C}$; au bout de 30 à 40 secondes la fréquence est inférieure à $20 \mathrm{~Hz}$, puis décline doucement jusqu'à l'immobilité totale (Cosson et al 1985 ; figure 3).

Tableau 3. Echelle d'appréciation de la motilité des spermatozoïdes de salmonidé observés au microscope, en contraste de phase, $G \times 160$, (d'après Sanchez-Rodriguez et Billard 1977).

\begin{tabular}{|c|c|}
\hline Valeur & Observations \\
\hline 5 & $\begin{array}{l}\text { - Tous les spermatozoïdes se } \\
\text { déplacent vigoureusement } \\
\text { impossibilité de fixer la vue sur } \\
\text { aucun d'entre eux. }\end{array}$ \\
\hline 4 & $\begin{array}{l}\text { - La majorité des spermatozoïdes } \\
\text { se déplace encore rapidement; } \\
\text { seuls quelques-uns sont visibles } \\
\text { du fait de leur déplacement plus } \\
\text { lent. }\end{array}$ \\
\hline 3 & $\begin{array}{l}\text { - Les spermatozoïdes présentent } \\
\text { trois comportements (en nombre } \\
\text { sensiblement égaux): } \\
\text { - soit ils se déplacent vigoureuse- } \\
\text { ment } \\
\text { - soit ils se déplacent lentement } \\
\text { - soit ils sont immobiles. }\end{array}$ \\
\hline 2 & $\begin{array}{l}\text { - Peu de spermatozoïdes se dépla- } \\
\text { cent rapidement ; quelques-uns } \\
\text { se déplacent lentement. La } \\
\text { majorité des spermatozoïdes est } \\
\text { immobile. }\end{array}$ \\
\hline 1 & $\begin{array}{l}\text { - Seuls quelques spermatozoïdes } \\
\text { ont une légère agitation. }\end{array}$ \\
\hline 0 & $\begin{array}{l}\text { Tous les spermatozoïdes sont } \\
\text { immobiles. }\end{array}$ \\
\hline
\end{tabular}

Figure 3. Evolution de la fréquence de battement du flagelle du spermatozoïde de truite en fonction du temps après l'activation (solution $125 \mathrm{mM} \mathrm{NaCl}, \mathrm{pH} 9,2$ ) à différentes températures (de $5^{\circ} \mathrm{C}$ à $25^{\circ} \mathrm{C}$ ) (d'après Cosson et al 1985); la fréquence initiale du battement et le taux d'inactivation pendant les 30 premières secondes dépendent de la température.

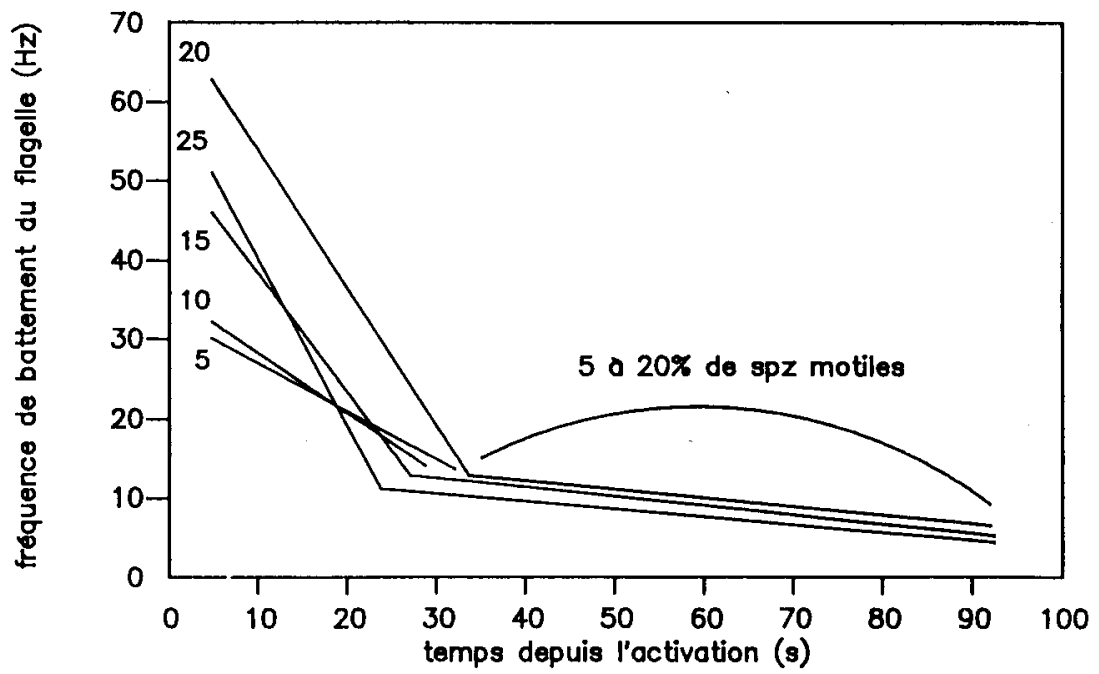

Après libération dans l'eau, le spermatozoïde de truite devient mobile pendant environ 30 secondes. 


\section{b / Physiologie de la motilité}

Chez les poissons marins, c'est le passage dans un milieu hyperosmotique (> $300 \mathrm{mOs}-$ moles) qui provoque la mise en mouvement des spermatozoïdes ; c'est au contraire le passage dans un milieu hypoosmotique qui active les spermatozoïdes des poissons d'eau douce comme la carpe. Pour les salmonidés, c'est la dilution du potassium présent dans le fluide séminal, qui entraîne l'activation ; l'inhibition de la motilité en présence de $\mathrm{K}^{+}$peut être levée par l'adjonction de $\mathrm{Ca}^{++}$(Baynes et al 1981, Tanimoto et Morisawa 1988). Utilisant des bloqueurs des canaux calciques et potassiques Tanimoto et Morisawa (1988) ont montré que l'activation de la motilité est initiée par une fuite de $\mathrm{K}^{+}$et une pénétration de $\mathrm{Ca}^{++}$. Ce phé-

Figure 4. Evolution de l'AMP cyclique intra-cellulaire dans les secondes suivant l'immersion de sperme de truite dans deux solutions, l'une à $100 \mathrm{mM} \mathrm{NaCl}$, provoquant l'activation, l'autre à $100 \mathrm{mM} \mathrm{KCl}$, maintenant l'immobilité des spermatozoides (d'après Morisawa et Ishida 1987). Lors de l'activation on observe une rapide augmentation de l'AMPC, le pic étant atteint en 1 seconde.

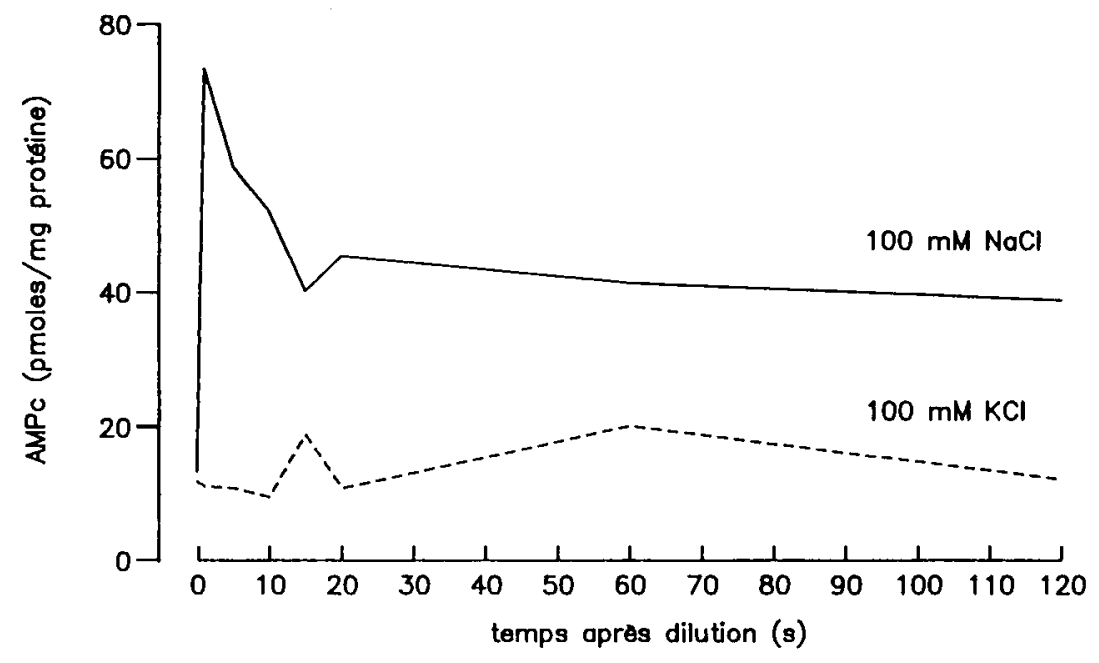

Figure 5. Evolution de l'ATP intra-cellulaire dans les minutes suivant limmersion de sperme de truite dans deux solutions, l'une à $125 \mathrm{mM} \mathrm{NaCl}$, provoquant l'activation, l'autre à 83 $m M ~ N a C l+42 \mathrm{mM} \mathrm{KCl}$, maintenant l'immobilité des spermatozoïdes (d'après Christen et al 1987). Lors de l'activation on observe une chute brutale (dans les 30 premières secondes) du niveau d'ATP et au bout d'une minute les spermatozoides sont immobilisés. La production d'ATP par la mitochondrie permet de retrouver le niveau initial au bout de 15 minutes; à ce moment les spermatozoides peuvent être réactivés en ajoutant au milieu $30 \mathrm{mM}$ de Ca2+.

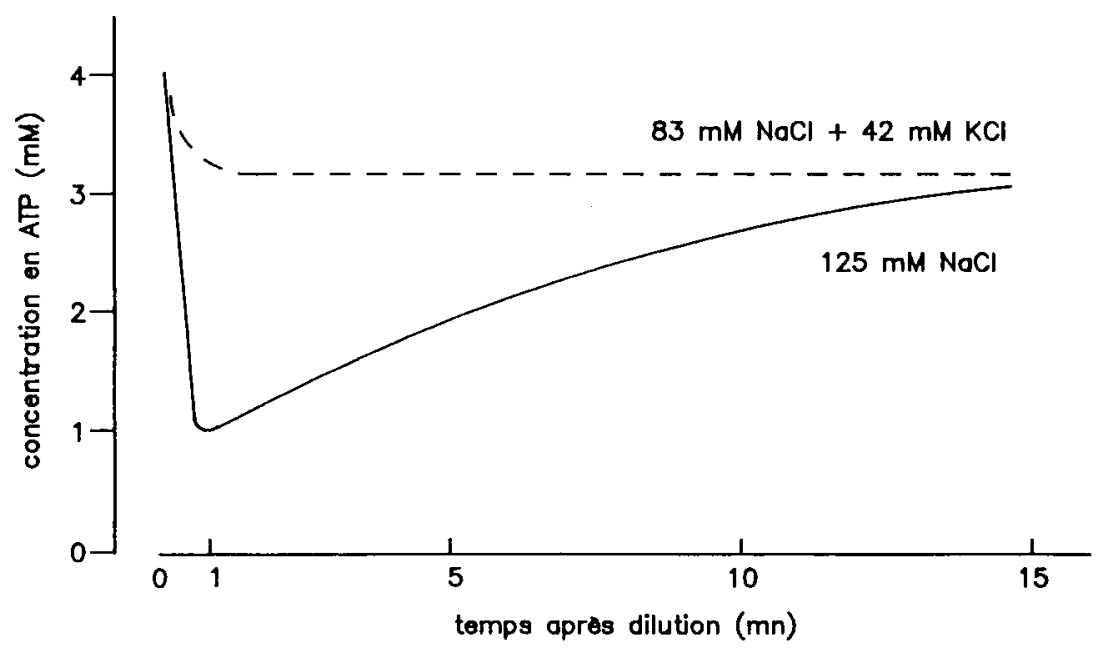

nomène activerait la transformation de l'ATP en AMP-cyclique dans la seconde suivant la libération du sperme dans l'eau (Morisawa et Ishida 1987, figure 4). L'AMP-c endogène jouerait alors un rôle dans l'initiation de la motilité (Benau et Terner 1980, Billard 1980, Morisawa et Okuno 1982), par l'activation d'une enzyme, la protéine-kinase, catalysant la phosphorylation d'une protéine (15 KDa) présente dans l'axonème ; cette protéine phosphorylée activerait le flagelle par un mécanisme non élucidé (Morisawa 1985). Par ailleurs, le Magnésium (5 à $10 \mathrm{mM}$ ) joue aussi un rôle dans le mouvement des spermatozoïdes (Baynes et al 1981); les ondulations du flagelle résultent du glissement les uns sur les autres des microtubules externes ; ce phénomène est provoqué par l'activité enzymatique (hydrolyse de $\mathrm{MgATP}^{2-}$ ) de la dynéine $19 S$ (protéine de grande taille, 1250 KDa) du bras externe des microtubules en doublet, maximum à pH 9 (Gatti et al 1989).

La durée d'activité extrèmement courte des spermatozoïdes de salmonidés dans un milieu isoosmotique (260 mOsmoles) est due à l'incapacité de la mitochondrie à compenser rapidement les pertes en ATP : en $30 \mathrm{~s}$ le niveau d'ATP chute de $3-4 \mathrm{mM}$ à $1 \mathrm{mM}$, déficit qui ne sera comblé qu'au bout de 15 minutes par l'activité de la mitochondrie ; la motilité des spermatozoïdes peut alors être réactivée par l'adjonction de $30 \mathrm{mM}$ de $\mathrm{Ca}^{++}$(Christen et al 1987, figure 5).

Les $\mathrm{pH}$ faibles sont nuisibles à l'activation des spermatozoïdes (Baynes et al 1981) et l'optimum pour la durée de motilité est 9 (Billard et Cosson 1988).

\section{4 / Application à la salmoniculture}

\section{1 / La fécondation artificielle}

A l'origine, la fécondation artificielle se pratiquait dans de l'eau de source dont la composition ionique et le $\mathrm{pH}$ varient suivant les lieux ce qui entraînait des résultats variables. Par ailleurs, le choc osmotique infligé aux spermatozoides dans ces conditions provoque rapidement un gonflement puis un éclatement de la membrane.

Billard (1977) propose un dilueur à base de $\mathrm{NaCl}(125 \mathrm{mM})$ ajustant la pression osmotique à 250 mOsmoles et tamponné (système TrisGlycocolle) à $\mathrm{pH} 9$, $\mathrm{pH}$ optimum pour la fécondation avec de forte dilutions (Petit et al 1973). Ce dilueur (DIA 532) permet de diluer le sperme au millième $(2 \mathrm{l}$ d'oeufs $+1 \mathrm{l}$ de dilueur + $1 \mathrm{ml}$ de sperme), tout en maintenant des niveaux de fécondation très élevés (environ 85 $\%$ en moyenne en pisciculture).

\section{2 / La conservation du sperme à court terme}

Nous avons vu que les spermatozoïdes, même immobiles, consomment de l'oxygène en quantité non négligeable. Billard et Legendre (1982) ont montré que, à $9^{\circ} \mathrm{C}$, la teneur initiale du sperme en oxygène $(6 \mathrm{mg} / \mathrm{l})$ chute brutalement de moitié au bout de 6 h lorsqu'il est 
conservé en présence d'air, alors qu'il se maintient au moins $24 \mathrm{~h}$ sous atmosphère d'oxygène. La conservation du sperme à des températures voisines de $0^{\circ} \mathrm{C}$ est grandement améliorée lorsqu'on le stocke en faible épaisseur sous atmosphère d'oxygène pur (Stoss 1983). Dans ces conditions, Stoss et Holtz (1983a) ont conservé une fécondance satisfaisante pendant 20 jours en supplémentant le sperme avec de la pénicilline et de la streptomycine.

\section{3 / Le prélèvement et l'utilisation de sperme testiculaire}

L'apparition dans les années 80 d'un nouveau produit, la truite de grande taille, a entraîné la nécessité d'élever des populations «monosexe femelle» ne présentant pas les inconvénients d'une maturation précoce. L'obtention de telles populations se fait par insémination artificielle classique d'œufs normaux, par du sperme de néomâles, génétiquement femelles. Ces néomâles, produits d'une inversion sexuelle artificielle (Methyltestostérone par voie orale pendant les 3 premiers mois d'alimentation), présentent fréquemment des malformations du canal déférent, obligeant le sacrifice du géniteur pour la récolte du sperme. Les testicules sont alors dilacérés dans une solution saline renfermant du potassium pour maintenir l'immobilité ; le MMLS proposé par Billard et Jalabert (1974) sur le modèle du fluide séminal peut être utilisé : $\mathrm{NaCl} 100 \mathrm{mM}$, $\mathrm{KCl} 28,3 \mathrm{mM}, \mathrm{MgSO}_{4} 1,1 \mathrm{mM}, \mathrm{CaCl}_{2}$ 1,8 mM, pH 9 (Tampon Tris-HCl 0,02 M).

\section{4 / La cryoconservation du sperme}

La cryoconservation du sperme fait appel à des dilueurs qui doivent dans un premier temps assurer l'isoosmolarité avec le milieu intérieur et éviter la mise en mouvement des spermatozoïdes, et dans un deuxième temps, lors de la congélation proprement dite, réduire autant que possible la formation de cristaux de glace et assurer le maximum de protection à la membrane des spermatozoïdes. Le dilueur actuellement utilisé est celui de Mounib modifié par Legendre et Billard (1980) : Sucrose 125 $\mathrm{mM}$, Gluthation réduit $6,5 \mathrm{mM}$, Bicarbonate de Potassium $100 \mathrm{mM}$, DMSO $10 \%$, Jaune d'CEuf au Tellurite $10 \%$. Les résultats obtenus avec ce dilueur ( 1 vol. de sperme pour 3 vol. de dilueur ; congélation en boulettes de $100 \mu \mathrm{l}$ sur de la carboglace; conservation dans l'azote liquide) sont variables d'un éjaculat à l'autre (Legendre et Billard 1980, Stoss et Holtz 1983b, Maisse et al 1988) et dépendent de la qualité de la membrane des spermatozoïdes (Malejac et al 1990). Un certain nombre d'indicateurs a priori de l'aptitude à la congélation du sperme ont été proposés: absence de $42 \mathrm{KDa}$ dans le fluide séminal (Maisse et al 1988), pression osmotique séminale inférieure à 280 MOsmoles et comportement de la membrane des spermatozoïdes dans une solution hypotonique (pourcentage de cellules éclatées inférieur à $10 \%$ après 24 heures dans une solutionà $60 \mathrm{mOsmoles}$ ) (Malejac et al 1990). Mais les facteurs de cette qualité du sperme sont difficiles à définir avec probablement de nombreuses interactions, ce
Figure 6. Fécondance, après dilution au centième, du sperme de truite à l'état frais, après conservation, pendant 8 et 15 jours, à une température voisine de $0^{\circ} \mathrm{C}$, sous une atmosphère d'oxygène pur, (avec adjonction d'antibiotiques), (selon Stoss et Holtz 1983a) et après congélation (selon Legendre et Billard 1980) et conservation plusieurs mois dans l'azote liquide, (Maisse, Pinson et Loir, non publié). D'un point de vue pratique, seule la conservation pendant 8 jours à une température voisine de $0^{\circ} \mathrm{C}$ est opérationnelle; la cryoconservation avec une performance moyenne de $40 \%$ de fécondation doit être améliorée pour devenir intéressante pour l'éleveur.

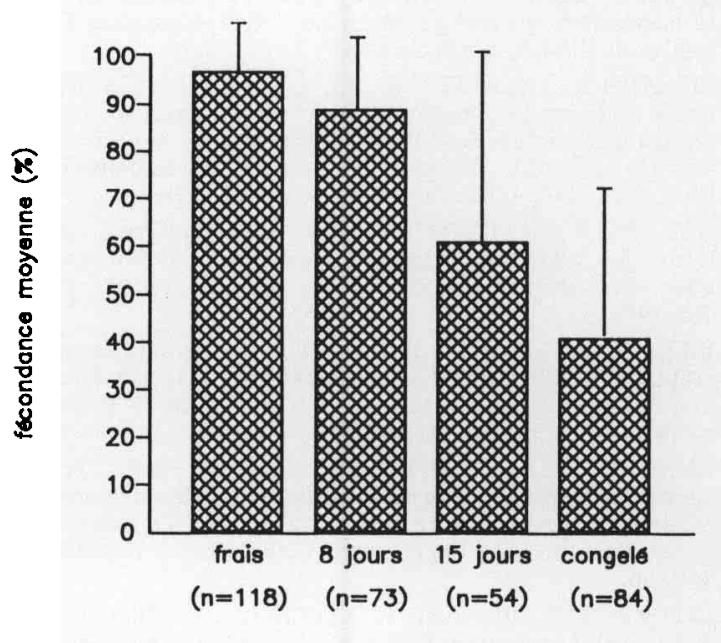

qui amène les auteurs à recommander le mélange de plusieurs spermes (Legendre et Billard 1980).

\section{Conclusion}

Les connaissances actuellement disponibles sur le sperme de salmonidés sont suffisantes à une gestion standardisée de l'insémination artificielle (prélèvement du sperme, conservation à court terme, fécondation). Cependant la particularité de la ponte (chaque femelle ne pond naturellement qu'une fois par an) rend l'utilisation de sperme congelé peu attrayante pour l'éleveur en dessous d'une fécondance de $75 \%$. Ce chiffre n'est pas encore atteint dans les conditions d'exploitation (figure 6) et c'est dans ce secteur que se situe le principal besoin de recherche finalisée sur le sperme. Nos récents travaux ont confirmé que l'aptitude à la congélation dépend de la qualité de la membrane des spermatozoïdes ; c'est dans cette direction que les recherches menées au Laboratoire de Physiologie des Poissons de l'INRA s'orientent, en étudiant plus particulièrement ce qui dans la spermatogénèse peut intervenir sur la composition lipidique de la membrane des spermatozoïdes (nutrition, température d'élevage...).

\section{Références bibliographiques}

BAYNES S.M., SCOT'T A.P., DAWSON A.P., 1981. Rainbow trout, Salmo gairdnerii Richardson, spermatozoa: effects of cations and pH on motility. J. Fish Biol., 19, 259-267.

BENAU D., TERNER C., 1980. Initiation, prolongation, and reactivation of the motility of salmonid spermatozoa. Gamete Res., 3, 247-257.
Des dilueurs ont été mis au point pour l'insémination artificielle, la manipulation de sperme testiculaire et la congélation. 
BILLARD R., 1977. Utilisation d'un système TRIS-Glycocolle pour tamponner le dilueur d'insémination de la truite. Bull. Fr. Pisc. 250, 17-34.

BILLARD R., 1980. Prolongation de la durée de motilité et du pouvoir fécondant des spermatozoïdes de truite arcen-ciel par addition de théophylline au milieu de dilution. C. R. Acad. Sc. Paris, 291, 649-652.

BILLARD R., 1983. Ultrastructure of trout spermatozoa Changes after dilution and deep-freezing. Cell. Tissue Res., 228, 205-218.

BILLARD R. COSSON M.P, 1988. Sperm motility in rainbow trout, Parasalmo mykiss ; effect of $\mathrm{pH}$ and temperature. Reproduction in fish - Basic and applied aspect in endocrinology and genetics. Ed. INRA, Paris (Les Col loques de l'INRA, n"44), 161-167.

BILLARD R., JALABERT B., 1974. L'insémination artificielle de la truite (Salmo gairdneri Richardson) II - Comparaison des effets de différents dilueurs sur la conservation de la fertilité des gamètes avant et après insémination. Ann. Biol. anim. Bioch. Biophys., 14, 601-610.

BILLARD R., LEGENDRE M., 1982. Conservation à court terme des gamètes de truite arc-en-ciel en condition in vitro sous atmosphère d'oxygène. Bull. Fr. Piscic., 284 162-167.

BILLARD R., MENEZO Y., 1984. The amino acid composilion of rainbow trout (Salmo gairdneri) seminal fluid and blood plasma: a comparison with carp (Cyprinus carpio). Aquaculture, 41, 255-258.

CHRISTEN R., GATTI J.L., BILLARD R., 1987. Trout sperm motility : the transient movement of trout sperm is related to changes in the concentration of ATP following the activation of the flagellar movement. Eur. J. Biochem. $166,667-671$

COSSON M.P., BILLARD R., GATTI J.L., CHRISTEN R. 1985. Rapid and quantitative assessment of trout spermatozoa motility using stroboscopy. Aquaculture, 46, 71-75.

COSTE M., 1856. Instructiuns pratiques sur la pisciculture. Masson Ed., Paris. 144 p.

GATTI J.L., COSSON M.P., CHRISTEN R., BILLARD R. LETELLIER L., 1985. Activation of trout sperm. In Fish Culture, 7th Conference, European Soc. Comp. Physiol Biochem., Barcelona, 26-28 aout 1985, A3-7.

GATTI J.L., KING S.M., MOSS A.G., WITMAN G.B. 1989. Outer arm dynein from trout spermatozoa; purification, polypeptide composition, and enzymatic properties. J. Biol. Chem., 264, 11450-11457.

HWANG P.C., IDLER D.R., 1969. A study of major cations, smotic pressure, and $\mathrm{pH}$ in seminal components of atlantic salmon. J. Fish. Res. Bd. Canada, 26, 413-419.

LEGENDRE M., BILLARD R., 1980. Cryopreservation of rainbow trout sperm by deep-freezing. Reprod. Nutr Dévelop., 20, 1859-1868.

LOIR M., LABBE C., MAISSE G., PINSON A., BOULARD G., MOUROT B. CHAMBEYRON F, 1990. Proteins of seminal fluid and spermatozoa in the trout (Oncorhynchus mykiss): partial characterization and variations Fish Physiol. Biochem., sous presse.

MAISSE G., PINSON A., LOIR M., 1988. Caractérisation de l'aptitude à la congélation du sperme de truite arc-enciel (Salmo gairdneri) par des critères physico-chimiques. Aquat. Living Resour., 1, 45-51.

MALEJAC M.L., LOIR M., MAISSE G., 1990. Qualité de la membrane des spermatozoïdes de truite arc-en-ciel (Oncorhynchus mykiss) ; relation avec l'aptitude du sperme à la congélation. Aquat. Living Resour. 3, 1-12. MARSHALL W.S., BRYSON S.E., IDLER D.R., 1989. Control of ion transport by the sperm duct epithelium of brook trout (Salvelinus fontinalis). Fish Physiol. Biochem., 7; 331-336.

MORISAWA M., 1985. Initiation mechanism of sperm motility at spawning in teleosts. Zool. Sci., 2, 605-615
MORISAWA M., OKUNO M., 1982. Cyclic AMP induces maturation of trout sperm axoneme to initiate motility. Nature, 285, 703-704.

MORISAWA M., ISHIDA K., 1987. Short-term changes in levels of cyclic AMP, Adenylate cyclase, and Phosphodiesterase during the initiation of sperm motility in rainbow trout. J. Exp. Biol,, 242, 199-204.

MUNKITTRICK K.R., MOCCLA R.D., 1987. Seasonal changes in the quality of rainbow trout (Salmo gairdneri) semen: effect of a delay in stripping on spermatocrit. motility, volume and seminal plasma constituents. Aquaculture, 64, 147-156

PETIT I., IALABERT $B$, CHEVASSUS B BILLARD $R$, 1973. L'insémination artificielle de la truite (Salmo gairdneri Richardson) I - Effets du taux de dilution, du $\mathrm{pH}$ et de la pression osmotique du dilueur sur la fécondation. Ann. Hydrobiol., 4, 201-210.

PIIRONEN J., 1985. Variation in the properties of milt from the finnish landlocked salmon (Salmo salar $\mathrm{m}$. Sebago Girard) during a spawning season. Aquaculture $48,337-350$.

ROBITAILLE PML, MUMFORD KG, BROWN GG. 1987. 31P nuclear magnetic resonance study of trout spermatozoa at rest, after motility, and during short-term storage. Biochem. Cell Biol., 65, 474-485.

SANCHEZ-RODRIGUEZ M., BILLARD R., 1977. Conservation de la motilité et du pouvoir fécondant du sperme de la truite arc-en-ciel maintenu à des températures voisines de $0^{\circ}$ C. Bull. Fr. Piscic., 265, 144-152.

STOSS J., 1983. Fish gamete preservation and spermatozoan physiology. In Fish physiology, Hoar W.S., Randa D.J., Donaldson E.M. Ed., vol. IXB, Academic Press, New York, 305-350.

STOSS J., HOLTZ W., 1983a. Successful storage of chilled rainbow trout (Salmo gairdneri) spermatozoa for up to 34 days. Aquaculture, 31, 269-274.

STOSS I HOLTZ W 1983b. Cryopreservation of rainbow trout (Salmo gairdneri) sperm : III. Effect of proteins in the diluent, sperm from different males and interval between sperm collection and freezing. Aquaculture, 31, 275-82.

TANIMOTO S., MORISAWA M., 1988. Roles for potassium and calcium channels in the initiation of sperm motility in rainbow trout. Develop. Growth \& Differ, 30 $117-124$.

\section{Summary}

\section{Salmonid milt : review and applications.}

Properties of salmonid milt are reviewed. The main peculiarities of the spermatozoa are : absence of acrosoma, immotility in seminal plasma due to the high level of potassium, activation following a dilution of environmental $\mathrm{K}^{+}$, short duration of motility due to a low mitochondrial capacity to maintain the concentration of ATP, and dependence on environmental conditions (temperature. $\mathrm{pH}$, oxygen).

In practice, these characteristics must be taken in account in salmonid sperm handling, short term storage and cryopreservation.

MAISSE G. Le sperme des salmonidés: le point sur les connaissances. Applications à la salmoniculture. INRA Prod. Anim., 3 (3), $223-228$ 